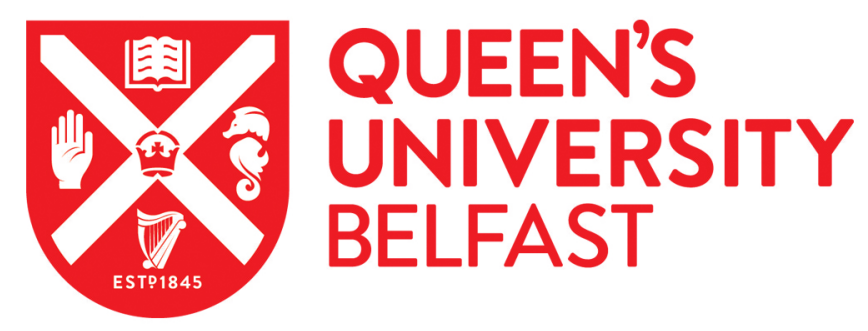

\title{
Factors that influence participation in physical activity for people with bipolar disorder: a synthesis of qualitative evidence
}

\author{
McCartan, C., Yap, J., Firth, J., Stubbs, B., Tully, M., Best, P., Webb, P., White, C., Gilbody, S., Churchill, R., \\ Breedvelt, J., \& Davidson, G. (2020). Factors that influence participation in physical activity for people with \\ bipolar disorder: a synthesis of qualitative evidence. Cochrane database of systematic reviews (Online), (3), \\ [CD013557]. https://doi.org/10.1002/14651858.CD013557
}

\section{Published in:}

Cochrane database of systematic reviews (Online)

\section{Document Version:}

Publisher's PDF, also known as Version of record

\section{Queen's University Belfast - Research Portal:}

Link to publication record in Queen's University Belfast Research Portal

\section{Publisher rights}

(C) 2020 The Cochrane Collaboration. Published by John Wiley \& Sons, Ltd. This work is made available online in accordance with the publisher's policies. Please refer to any applicable terms of use of the publisher.

\section{General rights}

Copyright for the publications made accessible via the Queen's University Belfast Research Portal is retained by the author(s) and / or other copyright owners and it is a condition of accessing these publications that users recognise and abide by the legal requirements associated with these rights.

\footnotetext{
Take down policy

The Research Portal is Queen's institutional repository that provides access to Queen's research output. Every effort has been made to ensure that content in the Research Portal does not infringe any person's rights, or applicable UK laws. If you discover content in the Research Portal that you believe breaches copyright or violates any law, please contact openaccess@qub.ac.uk.
} 


\section{Factors that influence participation in physical activity for people with bipolar disorder: a synthesis of qualitative evidence (Protocol)}

McCartan CJ, Yap J, Firth J, Stubbs B, Tully MA, Best P, Webb P, White C, Gilbody S, Churchill R, Breedvelt JJF, Davidson G

McCartan CJ, Yap J, Firth J, Stubbs B, Tully MA, Best P, Webb P, White C, Gilbody S, Churchill R, Breedvelt JJF, Davidson G. Factors that influence participation in physical activity for people with bipolar disorder: a synthesis of qualitative evidence. Cochrane Database of Systematic Reviews 2020, Issue 3. Art. No.: CD013557. DOI: 10.1002/14651858.CD013557.

www.cochranelibrary.com 
TABLE OF CONTENTS

HEADER 1

ABSTRACT

BACKGROUND

Figure 1.

OBJECTIVES

METHODS

ACKNOWLEDGEMENTS

REFERENCES

APPENDICES

CONTRIBUTIONS OF AUTHORS

DECLARATIONS OF INTEREST

SOURCES OF SUPPORT 
[Qualitative Protocol]

\section{Factors that influence participation in physical activity for people with bipolar disorder: a synthesis of qualitative evidence}

Claire J McCartan ${ }^{1}$, Jade Yap ${ }^{2}$, Joseph Firth ${ }^{3}$, Brendon Stubbs ${ }^{4}$, Mark A Tully 5 , Paul Best ${ }^{1}$, Paul Webb ${ }^{6}$, Chris White ${ }^{7}$, Simon Gilbody ${ }^{8}$, Rachel Churchill 9,10 , Josefien JF Breedvelt ${ }^{2}$, Gavin Davidson ${ }^{1}$

${ }^{1}$ Centre for Evidence \& Social Innovation, School of Social Sciences, Education \& Social Work, Queen's University Belfast, Belfast, UK. ${ }^{2}$ Mental Health Foundation, London, UK. ${ }^{3}$ Division of Psychology \& Mental Health, University of Manchester, Manchester, UK. ${ }^{4}$ Institute of Psychiatry, Psychology and Neuroscience, Kings College London, London, UK. 5 Institute of Mental Health Sciences, School of Health Sciences, Ulster University, Newtownabbey, UK. ${ }^{6}$ Praxis Care, Belfast, UK. ${ }^{7}$ Mental Health Foundation, Glasgow, UK. ${ }^{8}$ Mental Health and Addiction Research Group, Department of Health Sciences, University of York, York, UK. ${ }^{9}$ Centre for Reviews and Dissemination, University of York, York, UK. ${ }^{10}$ Cochrane Common Mental Disorders, University of York, York, UK

Contact address: Claire J McCartan, Centre for Evidence \& Social Innovation, School of Social Sciences, Education \& Social Work, Queen's University Belfast, 6 College Park, Belfast, Northern Ireland, BT7 1LP, UK. c.j.mccartan@qub.ac.uk.

Editorial group: Cochrane Common Mental Disorders Group.

Publication status and date: New, published in Issue 3, 2020.

Citation: McCartan CJ, Yap J, Firth J, Stubbs B, Tully MA, Best P, Webb P, White C, Gilbody S, Churchill R, Breedvelt JJF, Davidson G. Factors that influence participation in physical activity for people with bipolar disorder: a synthesis of qualitative evidence. Cochrane Database of Systematic Reviews 2020, Issue 3. Art. No.: CD013557. DOI: 10.1002/14651858.CD013557.

Copyright $\odot 2020$ The Cochrane Collaboration. Published by John Wiley \& Sons, Ltd.

\section{A B S T R A C T}

This is a protocol for a Cochrane Review (Qualitative). The objectives are as follows:

Main objective: To identify the factors that create barriers or facilitate physical activity for people with a diagnosis of bipolar disorder from the perspectives of service users, carers, service providers and practitioners to help inform the design and implementation of interventions that promote physical activity.

The overall aim of this review is to identify, appraise, and synthesise qualitative research evidence on the barriers and facilitators to engaging in physical activity in general lifestyle settings or as part of an intervention designed to increase physical activity for people with bipolar disorder. This will allow us to identify factors that create barriers and facilitators of physical activity in this population to inform the development, design, and implementation of future interventions. We will communicate our findings to public health commissioners and other stakeholders. 


\section{B A C K G R O U N D}

Physical activity is good for you but recent figures report that around 20 million adults (39\%) in the UK are physically inactive (BHF 2017). Worldwide, 1400 million people do not meet the weekly recommendations for physical activity established by the World Health Organization (WHO) (WHO 2010). Physical inactivity increases the risk of many adverse health conditions and is a significant cause of premature mortality (Lee 2012). Conservative estimates suggest that in 2013, physical inactivity cost healthcare systems USD 53,800 million worldwide, contributed to USD 13,700 million in loss in productivity and was responsible for GBP 13.4 million Disability-Adjusted Life Years (DALYs) (Ding 2016). Being physically active has the potential to protect against depression (Schuch 2018), and lower the risk of breast cancer, colon cancer, diabetes, ischaemic heart disease, and ischaemic stroke (Kyu 2016). Larger reductions in risks require significantly higher levels of physical activity than the minimum recommended level of 600 metabolic equivalent minutes per week (Kyu 2016). Recommended activity levels are higher for children and young people than adults (WHO 2012). High levels of sedentary behaviour in the youth population and the association with poor physical and mental health is a concern (Hoare 2016). Sedentary behaviour, independent of physical activity, has also been indicated in significant health risks including increased risk of diabetes, cardiovascular disease, and cardiovascular and all-cause mortality (Wilmot 2012).

Mental health problems contribute significantly to the overall disease burden worldwide and are major causes of disability, suicide, and ischaemic heart disease. People with mental ill health report lower levels of physical activity, and physical activity levels for those with bipolar disorder are considerably lower compared to the general population (Vancampfort 2016; Vancampfort 2017). People with bipolar disorder are at greater risk of chronic health conditions including cardiovascular disease and obesity, and many have co-occurring medical conditions (comorbid conditions) caused by lifestyle behaviours (e.g. smoking, hazardous substance misuse, sedentary behaviour) that can lead to poor health outcomes and impact negatively on their physical health (De Hert 2011; Kilbourne 2007; Osborn 2007; Roshanaei-Moghaddam 2009; (Sylvia 2011). People living with mental health conditions, including bipolar disorder, are also more likely to experience poverty (Elliott 2016). The multidimensional effects of poverty can have an influence on judgement and decision-making about important lifestyle behaviours, including personal choices about exercise and nutrition (Mullainathan 2014; Wilkinson 2018).

While there may be a range of social and genetic determinants of mental health disorders (Cross-Disorder Group 2013; WHO 2014), being physically active may have positive benefits and help improve the health of young people and adults with severe and enduring mental health conditions (Ströhle 2009). Based on secondary analysis of available data, physical activity has been shown to be potentially as effective as many drug interventions (e.g. statins, beta blockers) at preventing cardiovascular disease mortality (Naci 2013). However, evidence of the benefits of physical activity on mental health symptoms in bipolar disorder is limited (Stubbs 2018; Thomson 2015). Stubbs and colleagues' meta-review of physical activity as a treatment for severe mental illness (Stubbs 2018) identified two systematic reviews with people with bipolar disorder; one found some evidence that exercise may improve depressive symptoms and reduce stress but observed no changes in function, general symptoms or clinical global impression (de Sá Filho 2015), the second review reported weight loss in the treatment group compared to the control (Thomson 2015).

A number of barriers to physical activity are identified in the qualitative literature. They include low levels of motivation, poor self-perception, and inexperience/lack of competence (Cole 2010; Glowacki 2017). Practical issues such as cost (unemployment) and access (transport) can create barriers (Wynanden 2012). Aspects that help facilitate physical activity include the recognition of the psychological and physical benefits (Firth 2016), and good social and peer support networks (Schuch 2016). Gaining mastery and growing competency also encourages people to continue participation (Carless 2008; Firth 2016; Hodgson 2011). For young people, many of these barriers and facilitators are shared (e.g. cost, time, transport, infrastructure, social support, motivation), but additional barriers may be faced (Martins 2015). These can include negative associations with the competitive nature of physical activity within school and the pressure of winning/losing in front of their peer group or vested adults. Activity often reduces as children age (Dunmith 2011) and the transition periods, between primary to high school (10-12 years old) and high school to labour market entry (16-18 years old) (Bélanger 2011; Coleman 2008).

\section{Bipolar disorder}

Bipolar disorder consists of severe mood disturbances, neuropsychological deficits, and affects functioning (Rowland 2018). It is characterised by episodes of depression (feeling very low and lethargic) and mania (feeling very high and overactive). Symptoms can be diverse and will depend on whether a depressive or manic mood is being experienced. It can include compulsive behaviour, alcohol and substance misuse, and fluctuating energy levels (Hirschfield 2000). During periods of intense depression or mania, a person may experience psychotic symptoms that can lead to serious disturbances in their thinking, emotions, and behaviours. Each extreme episode can last for several weeks or longer and can interfere with everyday life. In 2017, there were 59.4 million cases of the disorder recorded, accounting for 121 million DALYs (Rehm 2019); it is associated with premature mortality from medical comorbidities and suicide. It may take some time for someone to be diagnosed correctly, but lifetime prevalence of bipolar disorder is estimated to affect $2.4 \%$ of the population (Merikangas 2011), and occurs in $1 \%$ to $3 \%$ of the youth population (Birmaher 2013). The mean age of onset is in the early 20s (Merikangas 2007), but several studies report that up to $60 \%$ of adult patients report the onset of their mood symptoms before the age of 21 (Chengappa 2003; Baldessarini 2010; Goodwin 2007). It appears to be equally distributed across both sex and ethnicity (Tsuchiya 2003), however presentation of the disorder may vary in patients with later-onset including higher rates of psychiatric and medical comorbidities (Cassidy 2002; Leboyer 2005). There are a number of risk factors for bipolar disorder including genetic and environmental factors (Craddock 1999; Craddock 2013; Marangoni 2016), but causation is difficult to establish (Rowland 2018). Environmental risk factors include stressful life events (Tsuchiya 2003), childhood maltreatment (Bortolato 2017; Garno 2005), and substance misuse (Post 2013). It is also associated with other medical conditions including inflammatory or stress-related disorders, such as irritable bowel syndrome (Bortolato 2017) and asthma (Wu 2016). 


\section{Description of the topic}

Physical activity is defined as any bodily movement produced by contraction of skeletal muscle that increases energy expenditure above a basal level (Caspersen 1985). This includes walking, cycling, sports, and other active forms of recreation (e.g. dance, gardening, yoga, tai chi). Exercise, a subcategory of physical activity is defined as planned, structured, and repetitive physical activity that is aimed at improving or maintaining one of more components of physical fitness (Caspersen 1985; Garber 2011; US Dept of HHS 2008). Physical inactivity is a global problem and understanding the reasons behind the motivation to be active forms part the WHO's 2018-2030 technical package to increase physical activity (WHO 2018). Activity rates are much lower for people with bipolar disorder than the general population (Vancampfort 2017). In a review and meta-analysis of sedentary behaviour and physical activity in schizophrenia, bipolar disorder, and major depressive disorder, people with bipolar disorder were the most active physically but were also significantly more sedentary $(615 \mathrm{~min}$ per day, $95 \% \mathrm{Cl} 456$ to $774 ; \mathrm{P}<0.001,12=99.2$ ) than those with schizophrenia (493 min per day, $95 \% \mathrm{Cl} 400$ to 586 ) or major depressive disorder (414 min per day, $95 \% \mathrm{Cl} 323$ to 505) (Vancampfort 2017). Although there is substantial evidence for the positive impact that physical activity has on mental health (Penedo 2005), there are few good-quality large-scale randomised trials in people with bipolar disorder (Stubbs 2018). Further exploration of the relationship between bipolar disorder and physical activity and its impact on mood symptoms is necessary (Thomson 2015), but given the high rate of medical co-morbidities, physical activity could potentially play an important role in both the prevention and treatment of physical health conditions.

Physical activity is relatively easy to deliver in home-based, clinical, or community settings, and carries a relatively low risk of negative side effects (Wright 2012); however, these can include that individuals' physical activity can become addictive, lead to 'muscle dysmorphia', associated risks with the use of anabolicandrogenic steroids, intense exercise and mood disturbances and overtraining syndrome (Peluso 2005). A number of methodological shortcomings in randomised controlled trials have made it difficult to assess how effective it is in a clinical setting (Ellis 2007; Faulkner 1999; Thomson 2015). The overall health benefits of being more active can have a positive impact on quality of life and well-being. Physical activity is increasingly used in consultant, allied health professional, and general practitioner referrals as a treatment option or as a complementary therapy (Price 2018), and exercise referral schemes are outlined in public health guidance by the National Institute for Health and Care Excellence (NICE) and recommended as treatment by the European Psychiatric Association (EPA) (NICE 2014; Stubbs 2018).

Many factors can create barriers or facilitate physical activity. The barriers can be increased for people with bipolar disorder. Better understanding of what motivates or inhibits individuals to be physically active could help equip practitioners to work more effectively with this client group (Wheeler 2018), and help to establish realistic physical activity goals within a treatment programme that do not exacerbate symptoms (Ehrlich 2015; Young 2017). This QES will synthesise the qualitative evidence for the factors that create barriers or facilitators of physical activity for people with bipolar disorder and outline what helps people with lived experience of this condition to be more active to benefit their mental health. It will complement another QES that will consider the facilitators and barriers for people with anxiety and depression (McCartan 2020).

\section{Why is it important to do this synthesis?}

Very low levels of physical activity remain a public health concern. Understanding more about the factors that create barriers or facilitate physical activity could help inform the design and development of interventions to improve the physical and mental health of young people and adults with bipolar disorder. This qualitative evidence synthesis (QES) will use the Health Belief Model (HBM) to examine these factors and outline what may help people with lived experience of bipolar disorder to be more physically active. The HBM is a theory of behaviour change developed to explain and help predict health-related behaviours, particularly related to the uptake of health services. The HMB has been used as a conceptual framework in health behaviour research since the 1950s to explore why people fail to participate in programmes designed to prevent and detect disease (Glanz 2010; Hochbaum 1958; Rosenstock 1960; Rosenstock 1974). It can help explain the change and maintenance of health-related behaviours and has been used as a guiding framework for many health-behaviour interventions (Jones 2014). This QES will examine how factors such as age, gender, class, ethnicity, mental health diagnosis, and co-morbid conditions affect participation. It will consider people's perceptions about their physical and mental health needs and identify barriers and facilitators to physical activity. It will also consider which cues influence people to take action. It will seek to understand why interventions work (or not), why people engage (or not) and how carers, service providers, and health professionals view, support, or influence treatment engagement.

A QES can help to identify the factors influencing the success of interventions including the attitudes and experiences of service users, their carers, service providers, and health professionals involved in their care. Two systematic reviews of exercise interventions for bipolar disorder concluded that lifestyle interventions including a physical activity component can be acceptable and efficacious for bipolar disorder (Bauer 2016; Melo 2016). These systematic reviews draw evidence from measures including quality of life, changes in depressive symptoms, and increased functioning but this evidence tells us little about the motivations, barriers, and facilitators to engaging in physical activity. Firth and colleagues conducted a systematic review and meta-analysis of quantitative data on the motivating factors and barriers towards exercise in serious mental illness (SMI) (Firth 2016). However, less is known about findings from qualitative studies. Developing a more nuanced understanding of how accessible physical activity is for people with bipolar disorder could bring important knowledge to the design, development, and implementation of interventions. QES provides the opportunity to add rich and contextual data and contribute to the knowledge base to help understand why and how best to increase physical activity levels among people with bipolar disorder without exacerbating symptoms. It will provide a greater insight into the factors that create barriers and facilitators of physical activity by exploring the lived experience of service users, carers, service providers, and practitioners to help highlight common beliefs, misconceptions and fears, and benefits of physical activity participation. 


\section{How the intervention might work}

Physical activity and exercise may help to reduce mental health symptoms for bipolar disorder (Kucyi 2010), but the evidence is limited (Stubbs 2018; Thomson 2015), and the cause and effect relationship has not been clearly established (Bauer 2016). However, being active may improve functioning and reduce depressive symptoms (Hayes 2017; Sylvia 2013a; Sylvia 2013b), and stimulate neurogenesis, metabolism, and vascular function (Cotman 2007; Ernst 2006). Physical activity may increase feelings of wellbeing by stimulating the release of endorphins (Steinberg 1985), and for those with mental health problems can reduce fatigue, improve sleep, and help with insomnia (Herring 2015; Rosenbaum 2015; Strid 2016). Being physically active can also contribute to physical changes (Bartels 2018), which in turn can boost self-esteem and positive perceptions of oneself. Physical activity can help deliver positive social experiences (Carless 2008); group-based activities can extend social networks and support systems for people who may have felt isolated because of their illness and feelings of low self-worth. Being active has also been linked to increased ratings of quality of life, pleasure, and motivation (Bauer 2016; Melo 2016). Evidence has also been reported for its effectiveness as an adjunct therapy to therapeutic interventions such as cognitive behaviour therapy for bipolar disorder (Sylvia 2013b).

Increasing physical activity to improve the health of people with bipolar disorder will often require specific behavioural changes (such as a decrease in sedentary behaviour and an increase in physical exertion). We want to understand how people with bipolar disorder adopt or reject positive physical activity behaviours. Applying a theoretical model of behaviour change can help understand the sequence of these mechanisms and identify what factors trigger positive change. This will identify which physical activity interventions are likely to be effective; to date, insufficient attention has been given to analysing behaviour change theories as a starting point for developing an intervention (Johnston 2008).

The HBM consists of six key concepts that predict why people will take action to prevent, screen for, or control illness conditions (Champion 2008), and acknowledges the range of modifying variables such as demographic, socio-economic, and structural factors that may also influence behaviour. It has been validated in a range of domains and populations (Carpenter 2010; Janz 1984). Although this model has its critics (Orji 2012), it can be a useful way to frame the barriers and facilitators for physical activity in bipolar disorder. The theoretical model identifies six domains which categorise the potential threats and expectations of a health behaviour and consider the cues or stimuli that will lead to action. Four additional domains were developed by Orji 2012 to improve its predictive power: concern for appearance; consideration of future consequences, perceived important, and self-identity. The six key concepts are:

- Perceived susceptibility: the perceived risk of getting a condition or disease. This will include a person's awareness and acceptance of their mental health diagnosis and any comorbid physical health needs.

- Perceived severity: the probability that a person will change their behaviour to avoid the consequence depends on how serious a condition they consider the consequence to be. How serious is the physical health condition when mental health is often the primary diagnosis? Does the service user understand the physical health risks associated with their mental health condition?

- Perceived benefits: the perception of the good things that could happen from taking part in specific behaviours. What's in it for the participant? People don't want to give up something they enjoy if they don't get something in return.

- Perceived barriers: the perception of the difficulties and costs of performing behaviours. This could relate to both intrinsic (shame, stigma, perceived lack of ability, lack of appropriate skills and knowledge) and extrinsic factors (cost, equipment, impact of medication, co-morbid conditions).

- Cues to action: exposure to factors that lead to action - public health messages, relationships with healthcare professionals, support from social/family systems, physical health checks, reminders, telemonitoring.

- Self-efficacy: confidence in one's ability to take action. Low levels of motivation and self-esteem.

See Figure 1. 
Figure 1. Health Belief Model for participation in physical activity for people with bipolar disorder

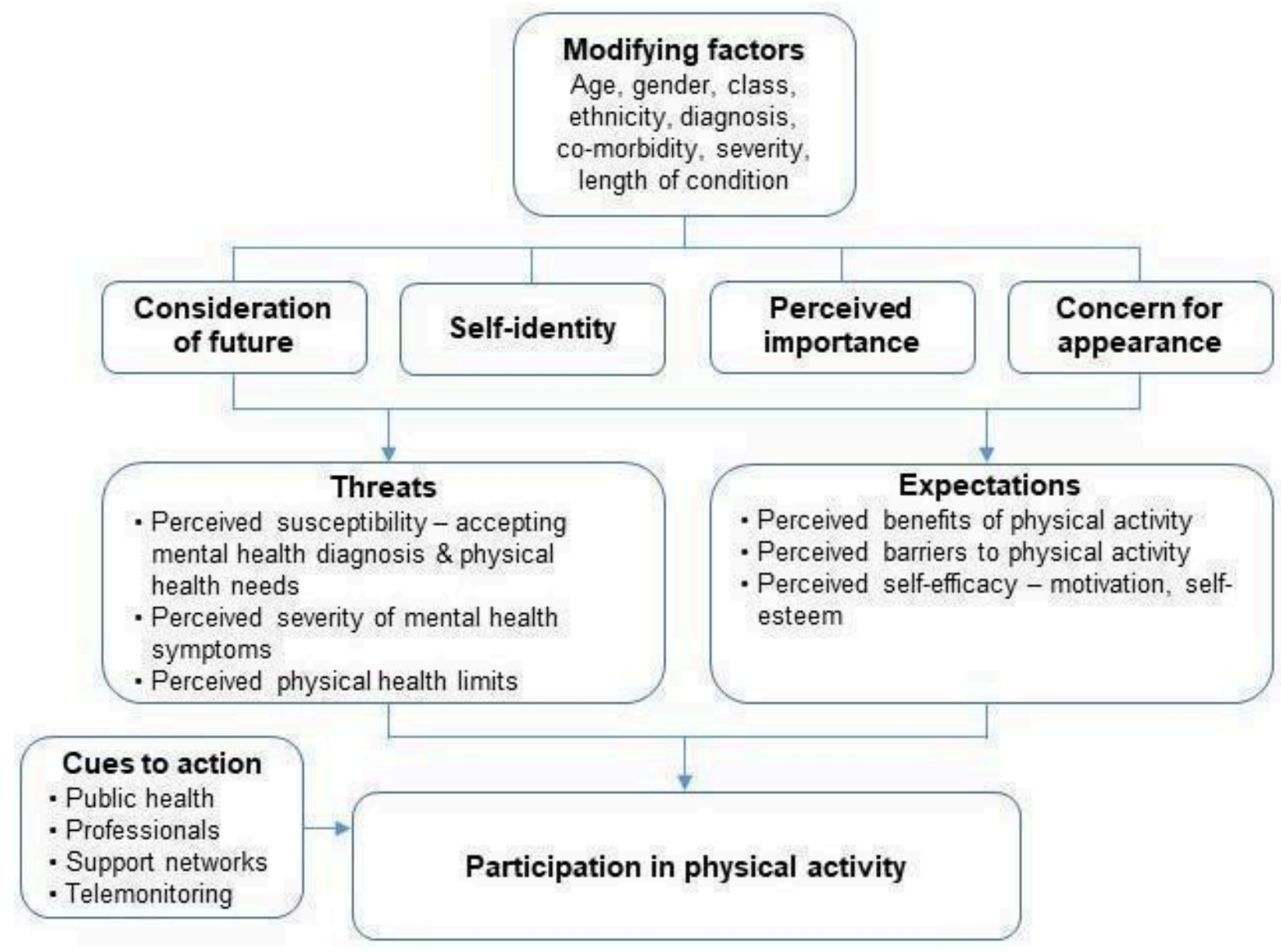

Perceived susceptibility and severity of physical health problems

Perceptions about the susceptibility and severity of physical health problems in bipolar disorder may be affected by current healthcare delivery. People with severe and enduring mental health problems may not be aware of all of their physical health needs - these can be neglected (Fagiolini 2009; Kisely 2007; Mitchell 2009; Tidemalm 2008) and there are inequalities in access to, use and delivery of healthcare in this population (Lawrence 2010; de Hert 2013). People with severe mental illness are less likely to receive health promotion interventions and other forms of preventive care such as routine cancer screening (Naylor 2016) and smoking cessation treatments (Gilbody 2019).

\section{Perceived benefits}

Improvements in general physical health, fitness, and body image and the psychological benefits of improved sleep, stress reduction, mood management, and increased self-confidence have been identified as perceived benefits (Firth 2016). Positive feelings associated with a sense of achievement, the acquisition of new skills, and developing mastery have also been cited in the literature (Carless 2008; Hodgson 2011; Wynanden 2012).

\section{Perceived barriers}

Many of the qualitative studies that consider the perceived barriers to engaging in physical activity cover intrinsic mechanisms that hinder involvement. These include low motivation or energy levels which can often be symptomatic of an illness (Cole 2010; Firth 2016), or sometimes a side effect of a particular medication or other health characteristics including high BMI or cigarette use (Osborn 2007). Psychological factors including embarrassment, poor perception of self, and guilt can also have a negative impact (Glowacki 2017), as well as general anxiety for social reasons, lack of experience, or perceived lack of ability or poor body image (Cole 2010; Firth 2016). Some studies also consider the counter effects of exercise participation and, where patients do not successfully initiate or maintain an exercise activity, this can have negative impact on their self-esteem (Searle 2012). Patient expectations of the impact of raised activity levels may also need to be managed (Jones 2005). Exercise may not have the desired affects for everyone, some studies have highlighted the potentially harmful risks for people with bipolar disorder during a manic episode (Wright 2012). Other issues to consider relate to practical difficulties including navigating public transport, high cost, and having no-one to accompany them (Wynanden 2012).

\section{Cues to action}

Cues to action, such as having appropriate social support systems in place can help encourage people to become more active and can also can have benefits beyond the intervention setting (Carless 
2008); it can help tackle participants' motivation and anxiety that may have prevented them from engaging in the past. This can be achieved over the phone or by other means such as telemonitoring as well as face-to-face contact (Argent 2018; Crone 2008; Searle 2014). Having someone to help familiarise participants with the setting and activities at the start of a programme can also be of great value (Firth 2016). Having peer support systems in place can help people engage (Firth 2016), and programmes that promote physical health, fitness, and body image outcomes can motivate involvement (Firth 2016).

\section{Self-efficacy}

Higher levels of self-efficacy evident in people who are already physically active or involved in sport are more likely to benefit (Dishman 1985), as will people who have higher levels of selfesteem, perceived physical condition, and body attractiveness.

\section{OB JECTIVES}

Main objective: To identify the factors that create barriers or facilitate physical activity for people with a diagnosis of bipolar disorder from the perspectives of service users, carers, service providers and practitioners to help inform the design and implementation of interventions that promote physical activity.

The overall aim of this review is to identify, appraise, and synthesise qualitative research evidence on the barriers and facilitators to engaging in physical activity in general lifestyle settings or as part of an intervention designed to increase physical activity for people with bipolar disorder. This will allow us to identify factors that create barriers and facilitators of physical activity in this population to inform the development, design, and implementation of future interventions. We will communicate our findings to public health commissioners and other stakeholders.

\section{METHODS}

\section{Review author reflexivity}

Throughout the data synthesis, the review authors will reflect on their own positions and assess how these could influence the study design, search strategy, inclusion decisions, data extraction, analysis, and synthesis, and interpretation of the findings. We are aware of our own professional backgrounds and one of the strengths of the team is the diversity of disciplines it includes (advocacy, mental health social work, psychiatry, physiotherapy, service user participation, service delivery, sports psychology), alongside clinical or research experience or both The reviewers have multiple perspectives but all support the principle that being physically active can have benefits for both physical and mental wellbeing and that intervention design and implementation strategies should be sensitive to the needs and experiences of service users, carers, service providers, and healthcare professionals. Progress will be discussed regularly and decisions that are made will be explored critically. As lead review author, $\mathrm{CMCC}$ will keep a reflexive journal throughout the process to document and reflect on progress and record discussions and decisions made. Stakeholder consultation will also provide additional opportunities to reflect and review author decisions.

\section{Criteria for considering studies for this review}

\section{Types of studies}

This is a systematic review of qualitative primary studies designed to increase physical activity participation to reduce the symptoms of bipolar disorder (reduction in symptoms). We will include primary studies that use qualitative study designs such as ethnography, phenomenology, case studies, grounded theory studies, and qualitative process evaluations. We will include studies that use both qualitative methods for data collection (e.g. focus group discussions, individual interviews, observation, diaries, document analysis, and open-ended survey questions) and qualitative methods for data analysis (e.g. thematic analysis, framework analysis, grounded theory). We will exclude studies that collect data using qualitative methods but do not analyse these data using qualitative analysis methods (e.g. open-ended survey questions where the response data are analysed using descriptive statistics only). We will include studies irrespective of their publication status and language of publication. Mixedmethods studies where qualitative findings can been extracted separately will also be included.

We will not exclude any studies based on our assessment of methodological limitations, but will utilise this information to assess our confidence in the synthesis findings.

\section{Topic of interest}

We will include studies that focus on the experiences and attitudes of service users, caregivers, service providers, and healthcare professionals towards physical activity for bipolar disorder. We will look for evidence of the factors that create barriers or facilitate participation in physical activity. We will include all approaches to increasing physical activity, supervised or unsupervised, in individual or group settings for people with bipolar for physical or mental health benefits. We will exclude yoga, tai chi, and other mind-body interventions because although these involve physical activity, the therapeutic benefits are theoretically derived from components of the intervention which are distinct from the physical activity itself. For instance, yoga is closely related to meditation and mindfulness, which share common practices and therapeutic principles grounded in religion spirituality that may offer benefits distinct from a purely physical activity intervention (Breedvelt 2019).

\section{Types of participants}

We will include studies where the primary focus is the experience of people with a primary clinical diagnosis of bipolar affective disorder (Diagnostic and Statistical Manual of Mental Disorders, 5th edition (DSM-5) (APA 2013)/International Statistical Classification of Diseases and Related Health Problems, 10th Revision (ICD-10) (WHO 1992)), their carers, service providers, and associated health professionals:

We will exclude mental health conditions that do not have a clinical diagnosis and age-related conditions (i.e. conditions or diseases that increase in incidence/prevalence with ageing such as dementia). We will include participants with other comorbid conditions, such as cardiovascular disease, stroke, and diabetes or other mental health conditions as long as there is a primary diagnosis of bipolar disorder. Studies of general severe mental illness cohorts will be included if individual 
patient comments can be identified as from individuals with bipolar disorder. We will include studies that provide qualitative evidence of the views and experiences of service providers, carers and healthcare professionals. Participants may be service users, paid (care assistants, home helps) or unpaid carers (including family members, friends), service providers (e.g. voluntary and community sector organisations delivering mental health services), and healthcare professionals (e.g. social workers, GPs, community mental health nurses, occupational therapists, physiotherapists, key workers, consultant psychiatrists).

\section{Search methods for identification of studies}

\section{Electronic searches}

We will develop search strategies for each database, using guidelines developed by the Cochrane Qualitative Research Methods Group for searching qualitative evidence (Harris 2018). We won't apply any restrictions on date, language, or publication status to the searches.

\section{Search terms}

\section{(i) Preliminary searches}

Identifying search terms for the intervention, those which address the barriers and facilitators to enable people with bipolar disorder to increase their levels of physical activity or start exercising, or both, are difficult to identify from the beginning.

We will perform an initial search on the following databases:

- Ovid MEDLINE (1946 onwards) (Appendix 1);

- Ovid PsycINFO (all years).

The preliminary searches will be based on population (bipolar disorder) combined with broad top-level terms for the intervention (barriers/facilitators) or a sensitive list of terms for outcome (physical activity).

Two review authors will screen the search results to identify relevant primary studies and reviews (quantitative and qualitative). They will screen the bibliographic records to identify title words, author assigned keywords, key phrases, search strings, and subject headings for the intervention. We will use these terms to enrich and refine the remaining searches (and if necessary amend the preliminary MEDLINE/PsycINFO searches, as appropriate).

\section{(ii) Main searches}

We will conduct additional searches on the following databases:

- Ovid Embase (1980 onwards);

- Wiley Cochrane Library (current issue);

- Web of Science Science Citation Index Expanded (SCIE) (all available years);

- Web of Science Social Science Citation Indexes (SSCI) (all available years);

- EBSCO Cumulative Index to Nursing and Allied Health Literature (CINAHL) (1980 onwards);

- ProQuest International Bibliography of Social Science (IBSS) (1951 onwards);

- ProQuest Sociological Abstracts (1952 onwards);

- EBSCO SPORTDiscus (1982 onwards);
- NCBI PubMed (not MEDLINE) subset (all years);

- Social Policy and Practice.

If any of these databases contain relatively few records for bipolar disorder, we will amend the structure of the search accordingly. Otherwise we will base the additional searches on the following structure: population (bipolar disorder) AND intervention (barriers or facilitators) AND outcome (physical activity).

\section{(iii) Searching other resources}

We will contact authors of included studies to clarify reported published information and to seek unpublished results/data. We will contact researchers with expertise relevant to the synthesis topic to request studies that might be eligible.

Once we have screened records and selected studies for inclusion, we will perform forward and backward citation searching. We will check the reference lists of all included studies and relevant systematic reviews to identify additional studies missed from the original electronic searches (e.g. unpublished or in-press citations). We will use the Web of Science and Google Scholar to conduct forward citation searching. We will also perform cluster searching (Booth 2013), and use PubMed's related references feature.

\section{(iv) Grey literature}

We will conduct a grey literature search to identify studies not indexed in the databases listed above.

- Open Grey;

- ProQuest Dissertations \& Theses Global;

- DART-Europe E-theses Portal (www.dart-europe.eu/);

- EThOS - the British Libraries e-theses online service (ethos.bl.uk/);

- Open Access Theses and Dissertations (oatd.org).

\section{Stakeholder involvement}

Stakeholders will be involved before, during, and after the review. We will draw on the expertise of an advisory group of people with lived experience and professionals working in the area, who will be involved in the problem framing stage and will be consulted to expand the search to include grey literature, relevant websites, and knowledge of programmes that have been delivered.

\section{Data collection, management, and synthesis}

\section{Selection of studies}

Records identified from different sources will be imported into EndNote and duplicates removed (EndNote 2019). Two review authors from a team of six (JB, PB, GD, CMcC, PW, JY) will independently screen the titles and abstracts of the retrieved records for their eligibility. We will retrieve the full text of all papers identified as potentially relevant by one or both review authors. Two review authors will then independently assess these papers. We will resolve any disagreements via discussion or, when required, by seeking a third review author's opinion. Where appropriate, we will contact the study authors for further information.

We will include a table listing the studies excluded from our synthesis at the full text stage and the main reasons for exclusion. 
Where the same study, using the same sample and methods, has been presented in different reports, we will collate these reports so that each study (rather than each report) is the unit of interest in the review.

We will include a PRISMA flow diagram to show our search results and the process of screening and selecting studies for inclusion.

\section{Translation of languages other than English}

For papers that are not published in a language that can be understood by the review authors (i.e. other than Dutch, French, German, Italian, Portuguese, Spanish), the abstract will be subject to initial translation through open source software (Google Translate). If this indicates inclusion, or if the translation is inadequate to make a decision, we will ask members of the multilingual networks associated with the research teams of the review to translate the full-text. If this cannot be done for a study in a particular language, we will list the study in the 'Studies awaiting classification' section, to ensure transparency in the review process.

\section{Sampling of studies}

We will select a sample of studies if more than 40 studies are eligible for inclusion. We will use a purposive sampling approach and apply maximum variation sampling with the aim of extracting the broadest possible variation within the eligible studies (Patton 2002). This approach has been demonstrated successfully in two other QES reviews (Ames 2017; Karimi-Shahanjarini 2019), and can help deal with large quantities of data that could potentially impair the quality of analysis (Glenton 2013). Included studies will be selected based on their direct relevance and which address the contextual factors important for the review question including the perspective, population, the phenomenon of interest and the setting. We will apply the GRADE-CERQual guidance to assess the contextual factors and relevance of the evidence to inform the sampling of studies (Noyes 2018).

\section{Data extraction}

Two review authors from a team of four (GD, CMCC, JY, PW) will independently extract data from each included study using a data extraction form (see Appendix 2). The data extraction template is based on the HMB, which includes specific information about: modifying factors (age, gender, socio-economic status, ethnicity, diagnosis, co-morbidity, severity or length of condition); perceived seriousness and susceptibility (consideration of future, self-identity, perceived importance, concern for appearance); threats; expectations; cues to action; and outcomes relating to participation in physical activity. We will record any theoretical frameworks used to analyse the data. Where additional themes are identified, these will be discussed by the review team. We will extract additional contextual and methodological information from the included studies detailing information about the country, study design, setting, population, participants, and a description of the intervention (if delivered) (logic model, number, frequency and duration of sessions). Methods used to identify and recruit the sample, data collection and analysis, relevant quantitative findings, supporting quotations, conclusions and any relevant tables, figures or images will be recorded. We will agree a final a priori framework using the Template for Intervention Description and Replication (TIDierR) checklist and guide (Hoffmann 2014) and we will code data from included studies against this framework.
Any disagreements will be resolved by discussion or by seeking the views of a third review author when necessary.

\section{Management and synthesis}

\section{Data synthesis}

We will use an adapted version of the HBM (Figure 1 ) as a basis for a 'best fit' framework synthesis (BFFS) approach to analyse and synthesise qualitative evidence (Booth 2015; Booth 2016; Carroll 2013). The HBM has been used to explore health beliefs and physical activity in a range of populations (Gristwood 2011; Hosseini 2017; Kasser 2012), and informed the development of physical activity interventions (Shao 2018). The selection of the HBM drew on the Cochrane Methods guidance for selecting a theory for complex intervention reviews (Noyes 2015); the model has the ability to consider complex interventions and has been validated and used in other systematic reviews. The review author team is also familiar with its application.

We chose the BFFS approach because it can be useful for designers of interventions, policymakers, and practitioners and is capable of describing and interpreting what is happening in a particular setting using a pragmatic approach to integrating data and engaging with existing theory (Booth 2016). It can be used to analyse and synthesise both primary and secondary data and to integrate quantitative and qualitative data (Booth 2015). However, should we deem the BFFS approach unsuitable as we begin to extract and code the data, we will use an alternative inductive thematic synthesis approach (Thomas 2008). BFFS requires the preliminary identification of a priori themes in order to map data from studies. Where any relevant data does not translate into the pre-existing constructs of the HBM, additional themes will be created as needed based on the emerging data. We will tailor the theoretical model to develop a 'best fit' theory (Noyes 2015). The HBM will provide a basis for the synthesis but will be 'builtupon, expanded upon, reduced or added to by these new data' as described by Carroll 2011 (p. 4). Data extraction and categorisation or coding of data will be discussed and agreed upon by the team at regular intervals. How modifying factors such as gender, race, and age will be discussed regularly to build the framework model. The BFFS approach will test the a priori framework and retest it using sensitivity analysis to explore how different modifying factors such as gender, race, age, and study quality affects the interpretation of the data (Booth 2015). It will also be important to capture the range of different perspectives (service user, carer, service provider, health professional) and assess whether an alternative explanation of engagement or non-engagement in physical activity is presented in the data and add this to the final model if appropriate. A consensus will be reached on the final list of themes.

The BFFS follows seven steps of synthesis, as follows:

1. identify a clearly-formulated review question

2. (a) systematically identify relevant primary research studies; and (b) identify relevant ('best fit') publications of frameworks, conceptual models or theories (e.g. HBM). We intend to use the $\mathrm{HBM}$ as our best fit framework. If this is not usable during data extraction, we will seek an alternative approach;

3. (a) extract data on study characteristics from included studies and appraise the quality of studies; and (b) generate the a priori framework from the modified HBM based on thematic analysis of the identified publication(s). We will extract data using a data 
extraction form. Study quality will be appraised using the CASP tool. We will generate an a priori framework from the analysis

4. code evidence from included studies against the a priori framework;

5. create new themes by performing secondary thematic analysis or thematic synthesis on any evidence that cannot be coded against the framework;

6. produce a new framework composed of a priori and new themes supported by the evidence; and

7. revisit evidence to explore relationships between themes or concepts, thus creating a model. 'Test' this synthesis and model by exploring the issues of dissonance and the impact of variables such as quality.

We will develop a 'Summary of qualitative findings' table from the synthesised findings once data extraction and analyses are completed.

\section{Assessment of the methodological limitations in included studies}

Two or more review authors (GD, CMcC, PW, JY) will independently assess methodological limitations for each study using the CASP Qualitative Checklist (www.casp-uk.net) to appraise the quality of included studies and the GRADE-CERQual. For studies where information is unclear or missing from reports, we will attempt to contact the study authors for further clarification. Any disagreements between review authors through discussion or by involving a third review author (PB, JF). We will assess methodological limitations according to the following domains:

- Are the results valid?

- Is a qualitative methodology appropriate?

- Was the research design appropriate to address the aims of the research?

- Was the recruitment strategy appropriate to the aims of the research?

- Was the data collected in a way that addressed the research issue?

- Has the relationship between researcher and participants been adequately considered?

- Have ethical issues been taken into consideration?

- Was the data analysis sufficiently rigorous?

- Is there a clear statement of findings?

- How valuable is the research?

We will report our assessment of methodological limitations for each study in the 'Characteristics of included studies' tables. A further assessment of methodological limitations of the body of data supporting each review finding will also be reported (MuntheKaas 2018).

\section{Assessment of heterogeneity}

A QES can be a valuable way of investigating the reasons why an intervention is effective (or not). Sensitivity analyses in the linked effectiveness reviews looked at intervention type and dose. We will also consider the variability in theory of change, outcomes, study designs, populations, interventions, and settings based on the evidence available. We will address clinical and methodological heterogeneity by including explicit and detailed methods for the identification, selection, and rating of the quality of individual studies and overall evidence. The 'best fit' framework analysis approach is more likely to make heterogeneity between studies and issues of quality appraisal more transparent (Barnett-Page 2009).

\section{Assessment of confidence in the synthesis findings}

We will use the CERQual (Confidence in the Evidence from Reviews of Qualitative research) approach to assess our confidence in the review findings (Colvin 2013; Glenton 2013; Lewin 2018; Munthe-Kaas 2018; Noyes 2018). This approach, building on the GRADE approach and the Cochrane tool for assessing risk of bias for Cochrane systematic reviews of interventions (GRADE Working Group 2004; Higgins 2017), is becoming the standard to assess confidence in the findings from qualitative evidence syntheses (Ames 2017; Bohren 2017; Colvin 2013; MunabiBabigumira 2017). Four review authors (GD, CMcC, JY, PW) will perform an independent assessment of the studies. Any disagreements will be discussed amongst review authors and an additional review author (PB, JF) consulted if necessary. The CERQual approach assesses the following four concepts (Lewin 2018).

1. Methodological limitations of included studies: the extent to which there are concerns about the design or conduct of the primary studies that contributed evidence to an individual review finding. Confidence in a finding may be lowered by substantial methodological limitations.

2. Coherence of the review finding: an assessment of how clear and cogent the fit is between the data from the primary studies and the review finding that synthesises the data. 'Cogent' refers to a well-supported or compelling fit. Variations in data across the included studies without convincing and cogent explanations may lower the confidence in a review finding.

3. Adequacy of the data contributing to a review finding: an overall determination of the degree of richness and quantity of data supporting a review finding. Confidence in a finding may be lowered if a finding is supported by results from only one or a few of the included studies, or when the data supporting a finding are very thin.

4. Relevance of the included studies to the review question: the extent to which the body of evidence from the primary studies supporting a review finding is applicable to the context (perspective or population, phenomenon of interest, setting) specified in the review question. Confidence in a finding may be lowered when contextual issues in a primary study used to support a review finding are different to the context of the review question.

After assessing each of the four components, we will make a judgement about the overall confidence in the evidence supporting the review finding. We will judge confidence as high, moderate, low, or very low. The final assessment will be based on consensus among the review authors. All findings start as high confidence and we will then downgrade them if there are important concerns regarding any of the CERQual components.

\section{'Summary of qualitative findings' table}

We will present findings in the 'Summary of qualitative findings' table, which will include an assessment of confidence in the evidence, as well as an explanation of this assessment, based on the GRADE-CERQual approach (Lewin 2018). 
Linking the synthesised qualitative findings to a Cochrane intervention review

There are no current Cochrane Reviews on the effectiveness of physical activity for bipolar disorder.

\section{ACKN OWLEDGEMENTS}

This review will inform research funded by the Disability Research on Independent Living and Learning (DRILL), which will involve the development of a co-produced physical activity intervention for people with lived experience of severe and enduring mental illness. $\mathrm{CMCC}$ was funded for 0.5 days per week over a three-month period to develop this protocol.

We thank the Cochrane Qualitative \& Implementation Methods Group for providing guidance during protocol development; and the editorial team of the Cochrane Common Mental Disorders (CCMD) Group including Sarah Dawson (Information Specialist) who helped develop the search strategies, Jessica Hendon and Sarah Hetrick.

The authors and the CCMD Editorial Team, are grateful to the peer reviewers for their time and comments including: Joseph Hayes, Jane Noyes, Danial Sayyad, and Abhijna Vithal Yergolkar. They would also like to thank copy editor, Deirdre Walshe.

Cochrane Group funding acknowledgement: The National Institute for Health Research (NIHR) is the largest single funder of the Cochrane Common Mental Disorders Group.

Disclaimer: The views and opinions expressed herein are those of the authors and do not necessarily reflect those of the NIHR, National Health Service (NHS), or the Department of Health and Social Care. 


\section{RE F E R E N C E S}

\section{Additional references}

\section{Ames 2017}

Ames HMR, Glenton C, Lewin S. Parents' and informal caregivers' views and experiences of communication about routine childhood vaccination: a synthesis of qualitative evidence. Cochrane Database of Systematic Reviews 2017, Issue 2. [DOI: 10.1002/14651858.CD011787.pub2]

\section{APA 2013}

American Psychiatric Association. Diagnostic and Statistical Manual of Mental Disorders. 5th Edition. Washington, DC: American Psychiatric Association, 2013.

\section{Argent 2018}

Argent R, Daly A, Caulfield B. Patient involvement with homebased exercise programs: can connected health interventions influence adherence?. JMIR Mhealth Uhealth 2018;6(3):e47. [DOI: 10.2196/mhealth.8518; 29496655; 5856927]

\section{Baldessarini 2010}

Baldessarini RJ, Bolzani L, Cruz N, Jones PB, Lai M, Lepri B, et al. Onset-age of bipolar disorders at six international sites. Journal of Affective Disorders 2010;121(1-2):143-6. [PUBMED: 19560827]

\section{Barnett-Page 2009}

Barnett-Page E, Thomas J. Methods for the synthesis of qualitative research: a critical review. BMC Medical Research Methodology 2009;9:59. [DOI: 10.1186/1471-2288-9-59]

\section{Bartels 2018}

Bartels SJ, Aschbrenner KA, Pratt SI, Naslund JA, Scherer EA, Zubkoff L, et al. Implementation of a lifestyle intervention for people with serious mental illness in state-funded mental health centers. Psychiatric Services 2018;69(6):664-70. [DOI: 10.1176/appi.ps.201700368; PUBMED: 29606077]

\section{Bauer 2016}

Bauer IE, Gálvez JF, Hamilton JE, Balanzá-Martínez V, ZuntaSoares GB, Soares JC, et al. Lifestyle interventions targeting dietary habits and exercise in bipolar disorder: a systematic review. Journal of Psychiatric Research 2016;74:1-7. [DOI: 10.1016/j.jpsychires.2015.12.006]

\section{BHF 2017}

British Heart Foundation. Physical inactivity and sedentary behaviour report 2017. Accessed at www.bhf.org.uk/ informationsupport/publications/statistics/physical-inactivityreport-2017 (accessed 23 January 2019).

\section{Birmaher 2013}

Birmaher B. Bipolar disorder in children and adolescents. Child and Adolescent Mental Health 2013;18(3):1-17. [DOI: 10.1111/ camh.12021]

\section{Bohren 2017}

Bohren MA, Hofmeyr GJ, Sakala C, Fukuzawa RK, Cuthbert A. Continuous support for women during childbirth. Cochrane
Database of Systematic Reviews 2017, Issue 7. [DOI: 10.1002/14651858.CD003766.pub6]

\section{Booth 2013}

Booth A, Harris J, Croot E, Springett J, Campbell F, Wilkins E. Towards a methodology for cluster searching to provide conceptual and contextual "richness" for systematic reviews of complex interventions: case study (CLUSTER). BMC Medical Research Methodology 2013;13:118.

\section{Booth 2015}

Booth A, Carroll C. How to build up the actionable knowledge base: the role of the 'best fit' framework synthesis for studies of improvement in healthcare. BMJ Quality \& Safety 2015;24(11):700-8.

\section{Booth 2016}

Booth A, Noyes J, Flemming K, Gerardus A, Wahlster P, Van Der Wilt GJ, et al. Guidance on choosing qualitative evidence synthesis methods for use in health technology assessments of complex interventions. 2016. www.integrate-hta.eu/wpcontent/uploads/2016/02/Guidance-on-choosing-qualitativeevidence-synthesis-methods-for-use-in-HTA-of-complexinterventions.pdf (accessed 28 January 2020).

\section{Bortolato 2017}

Bortolato B, Köhler CA, Evangelou E, Leon-Caballero J, Solmi M, Stubbs B, et al. Systematic assessment of environmental risk factors for bipolar disorder: an umbrella review of systematic reviews and meta-analyses. Bipolar Disorders 2017;19(2):84-96. [DOI: 10.1111/bdi.12490]

\section{Breedvelt 2019}

Breedvelt J, Amanvermez Y, Harrer M, Karyotaki E, Gilbody S, Bockting $\mathrm{C}$, et al. The effects of meditation, yoga, and mindfulness on depression, anxiety, and stress in tertiary education students: a meta-analysis. Frontiers in Psychiatry 2019;10:193. [DOI: 10.3389/fpsyt.2019.00193]

\section{Bélanger 2011}

Bélanger M, Casey M, Cormier M. Maintenance and decline of physical activity during adolescence: insights from a qualitative study. International Journal of Behavioral Nutrition and Physical Activity 2011;8:117. [DOI: 10.1186/1479-5868-8-117]

\section{Carless 2008}

Carless D, Douglas K. The contribution of exercise and sport to mental health promotion in serious mental illness: an interpretive project. International Journal of Mental Health Promotion 2008;10(4):5-12. [DOI: 10.1080/14623730.2008.9721771]

\section{Carpenter 2010}

Carpenter CJ. A meta-analysis of the effectiveness of health belief model variables in predicting behavior. Health Communication 2010;25(8):661-9. [DOI: $10.1080 / 10410236.2010 .521906]$ 


\section{Carroll 2011}

Carroll C, Booth A, Cooper K. A worked example of "best fit" framework synthesis: a systematic review of views concerning the taking of some potential chemopreventive agents. BMC Medical Research Methodology 2011;11:29. [DOI: 10.1186/1471-2288-11-29]

\section{Carroll 2013}

Carroll C, Booth A, Leaviss J, Rick J. "Best fit" framework synthesis: refining the method. BMC Medical Research Methodology 2013;13:37. [DOI: 10.1186/1471-2288-13-37]

\section{Caspersen 1985}

Caspersen CJ, Powell KE, Christenson GM. Physical activity, exercise, and physical fitness: definitions and distinctions for health-related research. Public Health Reports 1985;100(2):126-31. [PMC1424733; PUBMED: 3920711]

\section{Cassidy 2002}

Cassidy F, Carroll BJ. Vascular risk factors in late onset mania. Psychological Medicine 2002;32(2):359-62. [DOI: 10.1017/ S0033291701004718]

\section{Champion 2008}

Champion VL, Skinner CS. The health belief model. In: Glanz K, Rimer BK, Viswanath K editor(s). Health Behavior and Health Education. Theory, Research, and Practice. 4th Edition. San Francisco: Jossey-Bass, 2008:45-62.

\section{Chengappa 2003}

Chengappa KN, Kupfer DJ, Frank E, Houck PR, Grochocinski VJ, Cluss PA, et al. Relationship of birth cohort and early age at onset of illness in a bipolar disorder case registry. American Journal of Psychiatry 2003;160(9):1636-42. [PUBMED: 12944339]

\section{Cole 2010}

Cole F. Physical activity for its mental health benefits: conceptualising participation within the Model of Human Occupation. British Journal of Occupational Therapy 2010;73(12):607-15. [DOI: 10.4276/030802210X12918167234280]

\section{Coleman 2008}

Coleman L, Cox L, Roker D. Girls and young women's participation in physical activity: psychological and social influences. Health Education Research 2008;23(4):633-47. [DOI: 10.1093/her/cym040]

\section{Colvin 2013}

Colvin CJ, Heer J, Winterton L, Mellenkamp M, Glenton C, Noyes $\mathrm{J}$, et al. A systematic review of qualitative evidence on barriers and facilitators to the implementation of task-shifting in midwifery services. Midwifery 2013;29(10):1211-21. [DOI: 10.1016/j.midw.2013.05.001]

\section{Cotman 2007}

Cotman CW, Berchtold NC, Christie LA. Exercise builds brain health: key roles of growth factor cascades and inflammation. Trends in Neurosciences 2007;30(9):464-72. [DOI: 10.1016/ j.tins.2007.06.011; PUBMED: 17765329]

\section{Craddock 1999}

Craddock N, Jones I. Genetics of bipolar disorder. Journal of Medical Genetics 1999;36(8):585-94. [DOI: 10.1111/

j.1399-5618.2005.00181.x]

\section{Craddock 2013}

Craddock N, Sklar P. Genetics of bipolar disorder. Lancet 2013;381(9878):1654-62. [DOI: 10.1016/S0140-6736(13)60855-7]

\section{Crone 2008}

Crone D, Guy H. 'I know it is only exercise, but to me it is something that keeps me going': a qualitative approach to understanding mental health service users' experiences of sports therapy. International Journal of Mental Health Nursing 2008;17(3):197-207. [DOI: 10.1111/j.1447-0349.2008.00529.x; PUBMED: 18460081$]$

\section{Cross-Disorder Group 2013}

Cross-Disorder Group of the Psychiatric Genomics Consortium. Identification of risk loci with shared effects on five major psychiatric disorders: a genome-wide analysis. Lancet 2013;381(9875):1371-9. [DOI: 10.1016/S0140-6736(12)62129-1]

\section{De Hert 2011}

De Hert M, Correll CU, Bobes J, Cetkovich-Bakmas M, Cohen D, Asai I, et al. Physical illness in patients with severe mental disorders. I. Prevalence, impact of medications and disparities in health care. World Psychiatry 2013;10(1):52-77. [DOI: 10.1002/ j.2051-5545.2011.tb00014.x]

\section{de Hert 2013}

de Hert M, Correll CU, Bobes J, Cetkovich-Bakmas M, Cohen D, Asai I, et al. Physical illness in patients with severe mental disorders. I. Prevalence, impact of medications and disparities in health care. Work Psychiatry 2013;10(1):52-77. [DOI: 10.1002/ j.2051-5545.2011.tb00014.x]

\section{de Sá Filho 2015}

de Sá Filho AS, de Souza Moura AM, Lamego MK, Ferreira Rocha NB, Paes F, Oliveira C, et al. Potential therapeutic effects of physical exercise for bipolar disorder. CNS \& Neurological Disorders - Drug Targets 2015;14(10):1255-9. [DOI: 10.2174/1871527315666151111122219]

\section{Ding 2016}

Ding D, Lawson KD, Kolbe-Alexander TL, Finkelstein EA, Katzmarzyk PT, van Mechelen W, et al. Lancet Physical Activity Series 2 Executive Committee. The economic burden of physical inactivity: a global analysis of major non-communicable diseases. Lancet 2016;388(10051):1311-24. [DOI: 10.1016/ S0140-6736(16)30383-X]

\section{Dishman 1985}

Dishman RK, Sallis JF, Orenstein DR. The determinants of physical activity and exercise. Public Health Reports 1985;100(2):158-71. [MEDLINE: 3920714; PMC1424729]

\section{Dunmith 2011}

Dumith SC, Gigante DP, Domingues MR, Kohl HW 3rd. Physical activity change during adolescence: a systematic review 
and a pooled analysis. International Journal of Epidemiology 2011;40(3):685-98. [DOI: 10.1093/ije/dyq272]

\section{Ehrlich 2015}

Ehrlich C, Kendall E, Frey N, Denton M, Kisely S. Consensus building to improve the physical health of people with severe mental illness: a qualitative outcome mapping study. BMC Health Services Research 2015;15:83. [DOI: 10.1186/ s12913-015-0744-0; PMC4351836; PUBMED: 25879760]

\section{Elliott 2016}

Elliott I. Poverty and mental health. A review to inform the Joseph Rowntree Foundation's Anti-Poverty Strategy. www.mentalhealth.org.uk/sites/default/files/Poverty\%20and \%20Mental\%20Health.pdf (accessed 24 January 2019).

\section{Ellis 2007}

Ellis N, Crone D, Davey R, Grogan S. Exercise interventions as an adjunct therapy for psychosis: a critical review. British Journal of Clinical Psychology 2007;46(Pt 1):95-111. [PUBMED: 17472204]

\section{EndNote 2019 [Computer program]}

Clarivate Analytics. EndNote. Philadelphia: Clarivate Analytics, 2019.

\section{Ernst 2006}

Ernst C, Olson AK, Pinel JP, Lam RW, Christie BR. Antidepressant effects of exercise: evidence for an adult-neurogenesis hypothesis?. Journal of Psychiatry \& Neuroscience 2006;31(2):84-92. [PMC1413959; PUBMED: 16575423]

\section{Fagiolini 2009}

Fagiolini A, Goracci A. The effects of undertreated chronic mental illness in patients with severe mental disorders. Journal of Clinical Psychiatry 2009;70(Suppl 3):22-9. [DOI: 10.4088/ JCP.7075su1c.04]

\section{Faulkner 1999}

Faulkner G, Biddle S. Exercise as an adjunct treatment for schizophrenia: a review of the literature. Journal of Mental Health 1999;8(5):441-57. [DOI: 10.1080/09638239917157]

\section{Firth 2016}

Firth J, Rosenbaum S, Stubbs B, Gorczynski P, Yung AR, Vancampfort D. Motivating factors and barriers towards exercise in severe mental illness: a systematic review and meta-analysis. Psychological Medicine 2016;46(14):2869-81. [DOI: 10.1017/ S0033291716001732; PMC5080671; PUBMED: 27502153]

\section{Garber 2011}

Garber CE, Blissmer B, Deschenes MR, Franklin BA, Lamonte MJ, Lee IM, et al. American College of Sports Medicine. American College of Sports Medicine position stand. Quantity and quality of exercise for developing and maintaining cardiorespiratory, musculoskeletal, and neuromotor fitness in apparently healthy adults: guidance for prescribing exercise. Medicine \& Science in Sports \& Exercise 2011;43(7):1334-59. [DOI: 10.1249/ MSS.0b013e318213fefb; PUBMED: 21694556]

\section{Garno 2005}

Garno JL, Goldberg JF, Ramirez PM, Ritzler BA. Impact of childhood abuse on the clinical course of bipolar disorder. British Journal of Psychiatry 2005;186:121-5. [DOI: 10.1192/ bjp.186.2.121]

\section{Gilbody 2019}

Gilbody S, Peckham E, Bailey D, Arundel C, Heron P, Crosland S, et al. Smoking cessation for people with severe mental illness (SCIMITAR+): a pragmatic randomised controlled trial. The Lancet Psychiatry 2019;6(5):379-90. [DOI: 10.1016/ S2215-0366(19)30047-1]

\section{Glanz 2010}

Glanz K, Bishop DB. The role of behavioral science theory in development and implementation of public health interventions. Annual Review of Public Health 2010;31:399-418. [DOI: 10.1146/annurev.publhealth.012809.103604]

\section{Glenton 2013}

Glenton C, Colvin C, Carlsen B, Swartz A, Lewin S, Noyes J, et al. Barriers and facilitators to the implementation of lay health worker programmes to improve access to maternal and child health: qualitative evidence synthesis. Cochrane Database of Systematic Reviews 2013, Issue 10. [DOI: 10.1002/14651858.CD010414.pub2]

\section{Glowacki 2017}

Glowacki K, Duncan MJ, Gainforth H, Faulkner G. Barriers and facilitators to physical activity and exercise among adults with depression: a scoping review. Mental Health \& Physical Activity 2017;13:108-19. [DOI: 10.1016/j.mhpa.2017.10.001]

\section{Goodwin 2007}

Goodwin FK, Redfield Jamison K. Manic-Depressive Illness: Bipolar Disorders and Recurrent Depression. 2nd Edition. New York (NY): Oxford University Press, 2007.

\section{GRADE Working Group 2004}

GRADE Working Group. Grading quality of evidence and strength of recommendations. BMJ 2004;328:1490-4. [DOI: 10.1136/bmj.328.7454.1490]

\section{Gristwood 2011}

Gristwood J. Applying the Health Belief Model to physical activity engagement among older adults. Illuminare: $a$ Student Journal in Recreation, Parks, and Leisure Studies 2011;9(1):59-71.

\section{Harris 2018}

Harris JL, Booth A, Cargo M, Hannes K, Harden A, Flemming K, et al. Cochrane Qualitative and Implementation Methods Group guidance series-paper 2: methods for question formulation, searching, and protocol development for qualitative evidence synthesis. Journal of Clinical Epidemiology 2018;97:39-48. [DOI: 10.1016/j.jclinepi.2017.10.023; PUBMED: 29248725]

\section{Hayes 2017}

Hayes JF, Marston L, Walters K, King MB, Osborn DP. Mortality gap for people with bipolar disorder and schizophrenia: UKbased cohort study 2000-2014. British Journal of Psychiatry 
2017;211(3):175-81. [DOI: 10.1192/bjp.bp.117.202606; PMC5579328; PUBMED: 28684403]

\section{Herring 2015}

Herring MP, Kline CE, O'Connor PJ. Effects of exercise on sleep among young women with Generalized Anxiety Disorder. Mental Health and Physical Activity 2015;9:59-66.

\section{Higgins 2017}

Higgins JP, Altman DG, Sterne JA (editors). Chapter 8: Assessing risk of bias in included studies. In: Higgins JP, Churchill R, Chandler J, Cumpston MS, editor(s), Cochrane Handbook for Systematic Reviews of Interventions version 5.2.0 (updated June 2017). The Cochrane Collaboration, 2017. Available from www.training.cochrane.org/handbook. Version 5.2.0 (updated June 2017). Chichester: John Wiley \& Sons.

\section{Hirschfield 2000}

Hirschfeld RM, Williams JB, Spitzer RL, Calabrese JR, Flynn L, Keck PE Jr, et al. Development and validation of a screening instrument for bipolar spectrum disorder: the Mood Disorder Questionnaire. American Journal of Psychiatry 2000;157(11):1873-5. [DOI: 10.1176/appi.ajp.157.11.1873]

\section{Hoare 2016}

Hoare E, Milton K, Foster C, Allender S. The associations between sedentary behaviour and mental health among adolescents: a systematic review. International Journal of Behavioral Nutrition and Physical Activity 2016;13(1):108. [DOI: 10.1186/s12966-016-0432-4]

\section{Hochbaum 1958}

Hochbaum G. Public participation in medical screening programs: a sociopsychological study. Public Health Service Publication no. 572. Washington, DC: US Government Printing Office, 1958.

\section{Hodgson 2011}

Hodgson MH, McCulloch HP, Fox KR. The experiences of people with severe and enduring mental illness engaged in a physical activity programme integrated into the mental health service. Mental Health and Physical Activity 2011;4(1):23-9. [DOI: 10.1016/j.mhpa.2011.01.002]

\section{Hoffmann 2014}

Hoffmann TC, Glasziou PP, Boutron I, Milne R, Perera R, Moher D, et al. Better reporting of interventions: template for intervention description and replication (TIDierR) checklist and guide. BMJ 2014;348:g1687. [DOI: 10.1136/bmj.g1687]

\section{Hosseini 2017}

Hosseini H, Moradi R, Kazemi A, Sadat Shahshahani M. Determinants of physical activity in middle-aged women in Isfahan using the health belief model. Journal of Education and Health Promotion 2017;6:26. [DOI: 10.4103/jehp.jehp_68_15]

\section{Janz 1984}

Janz NK, Becker MH. The Health Belief Model: a decade later. Health Education Quarterly 1984;11(1):1-47. [DOI: $10.1177 / 109019818401100101]$

\section{Johnston 2008}

Johnston M, Dixon D. Current issues and new directions in psychology and health: what happened to behaviour in the decade of behaviour?. Psychology and Health 2008;23(5):509-13. [EMBASE: $10.1080 / 08870440701816728]$

\section{Jones 2005}

Jones F, Harris P, Waller H, Coggins A. Adherence to an exercise prescription scheme: the role of expectations, selfefficacy, stage of change and psychological well-being. British Journal of Health Psychology 2005;10(Pt 3):359-78. [DOI: 10.1348/135910704X24798; PUBMED: 16238853]

\section{Jones 2014}

Jones CJ, Smith H, Llewellyn C. Evaluating the effectiveness of health belief model interventions in improving adherence: a systematic review. Health Psychology Review 2014;8(3):253-69. [DOI: 10.1080/17437199.2013.802623]

\section{Karimi-Shahanjarini 2019}

Karimi-Shahanjarini A, Shakibazadeh E, Rashidian A, Hajimiri K, Glenton C, Noyes J, et al. Barriers and facilitators to the implementation of doctor-nurse substitution strategies in primary care: a qualitative evidence synthesis. Cochrane Database of Systematic Reviews 2019, Issue 4. [DOI: 10.1002/14651858.CD010412.pub2]

\section{Kasser 2012}

Kasser SL, Kosma M. Health beliefs and physical activity behavior in adults with multiple sclerosis. Disability Health Journal 2012;5(4):261-8. [DOI: 10.1016/j.dhjo.2012.07.001]

\section{Kilbourne 2007}

Kilbourne AM, Brar JS, Drayer RA, Xu X, Post EP. Cardiovascular disease and metabolic risk factors in male patients with schizophrenia, schizoaffective disorder, and bipolar disorder. Psychosomatics 2007;48(5):412-7. [DOI: 10.1176/ appi.psy.48.5.412]

\section{Kisely 2007}

Kisely S, Smith M, Lawrence D, Cox M, Campbell LA, Maaten S. Inequitable access for mentally ill patients to some medically necessary procedures. Canadian Medical Association Journal 2007;176(6):779-84. [PMC1808525]

\section{Kucyi 2010}

Kucyi A, Alsuwaidan MT, Liauw SS, McIntyre RS. Aerobic physical exercise as a possible treatment for neurocognitive dysfunction in bipolar disorder. Postgraduate Medicine 2010;122(6):107-16. [DOI: 10.3810/pgm.2010.11.2228; PUBMED: 21084787]

\section{Kyu 2016}

Kyu HH, Backman VF, Alexander LT, Mumford JE, Afshin A, Estep K, et al. Physical activity and risk of breast cancer, colon cancer, diabetes, ischemic heart disease, and ischemic stroke events: systematic review and dose-response meta-analysis for the Global Burden of Disease Study 2013. BMJ 2016;354:i3857. [DOI: 10.1136/bmj.i3857] 


\section{Lawrence 2010}

Lawrence D, Kisely S. Review: inequalities in healthcare provision for people with severe mental illness.

Journal of Psychopharmacology 2010;24(4):61-8. [DOI: 10.1177/1359786810382058; PUBMED: 20923921]

\section{Leboyer 2005}

Leboyer M, Henry C, Paillere-Martinot ML, Bellivier F. Age at onset in bipolar affective disorders: a review. Bipolar Disorders 2005;7(2):111-8. [DOI: 10.1111/j.1399-5618.2005.00181.x]

\section{Lee 2012}

Lee IM, Shiroma EJ, Lobelo F, Puska P, Blair SN, Katzmarzyk PT, Lancet Physical Activity Series Working Group. Effect of physical inactivity on major non-communicable diseases worldwide: an analysis of burden of disease and life expectancy. Lancet 2012;308(9838):219-29. [DOI: 10.1016/S0140-6736(12)61031-9; PUBMED: 22818936]

\section{Lewin 2018}

Lewin S, Booth A, Glenton C, Munthe-Kaas H, Rashidian A, Wainwright $\mathrm{M}$, et al. Applying GRADE-CERQual to qualitative evidence synthesis findings: introduction to the series. Implementation Science 2018;13(Suppl 1):2. [DOI: 10.1186/ s13012-017-0688-3]

\section{Marangoni 2016}

Marangoni C, Hernandez M, Faedda GL. The role of environmental exposures as risk factors for bipolar disorder: a systematic review of longitudinal studies. Journal of Affective Disorders 2016;193:165-74. [DOI: 10.1016/j.jad.2015.12.055]

\section{Martins 2015}

Martins J, Marques A, Sarmento H, Carreiro da Costa F. Adolescents' perspectives on the barriers and facilitators of physical activity: a systematic review of qualitative studies. Health Education Research 2015;30(5):742-55. [DOI: 10.1093/ her/cyv042]

\section{McCartan 2020}

McCartan C, Yap J, Firth J, Stubbs B, Tully M, Best P, et al. Factors that influence participation in physical activity for anxiety or depression: a synthesis of qualitative evidence. Cochrane Database of Systematic Reviews 2020, Issue 3. [DOI: 10.1002/14651858.CD013547]

\section{Melo 2016}

Melo MC, Daher ED, Albuquerque SG, de Bruin VM. Exercise in bipolar patients: a systematic review. Journal of Affective Disorders 2016;198:32-8. [DOI: 10.1016/j.jad.2016.03.004]

\section{Merikangas 2007}

Merikangas KR, Akiskal HS, Angst J, Greenberg PE, Hirschfield RM, Petukhova M, et al. Lifetime and 12-month prevalence of bipolar spectrum disorder in the National Comorbidity Survey replication. Archives of General Psychiatry 2007;64(5):543-52. [DOI: 10.1001/archpsyc.64.5.543]

\section{Merikangas 2011}

Merikangas KR, Jin R, He JP, Kessler RC, Lee S, Sampson NA, et al. Prevalence and correlates of bipolar spectrum disorder in the world mental health survey initiative. Archives of General Psychiatry 2011;68(3):241-51. [DOI: 10.1001/ archgenpsychiatry.2011.12]

\section{Mitchell 2009}

Mitchell AJ, Malone D, Doebbeling CC. Quality of medical care for people with and without comorbid mental illness and substance misuse: systematic review of comparative studies. British Journal of Psychiatry 2009;194(6):491-9. [DOI: 10.1192/ bjp.bp.107.045732]

\section{Mullainathan 2014}

Mullainathan S, Shafir E. Scarcity: the True Cost of Not Having Enough. London: Penguin Books, 2014.

\section{Munabi-Babigumira 2017}

Munabi-Babigumira S, Glenton C, Lewin S, Fretheim A, Nabudere $\mathrm{H}$. Factors that influence the provision of intrapartum and postnatal care by skilled birth attendants in low- and middle-income countries: a qualitative evidence synthesis. Cochrane Database of Systematic Reviews 2017, Issue 11. [DOI: 10.1002/14651858.CD011558.pub2]

\section{Munthe-Kaas 2018}

Munthe-Kass H, Bohren MA, Glenton C, Lewin S, Noyes J, Tunçalp Ö, et al. Applying GRADE-CERQual to qualitative evidence synthesis findings-paper 3: how to assess methodological limitations. Implementation Science 2018;13(Suppl 1):9. [DOI: 10.1186/s13012-017-0690-9]

\section{Naci 2013}

Naci $\mathrm{H}$, loannidis JP. Comparative effectiveness of exercise and drug interventions on mortality outcomes: metaepidemiological study. BMJ 2013;347:f5577. [DOI: 10.1136/ bmj.f5577]

\section{Naylor 2016}

Naylor C, Das P, Ross S, Honeyman M, Thompson J, Gilburt H. Bringing together physical and mental health: a new frontier for integrated care. London: King's Fund, 2016.

\section{NICE 2014}

National Institute for Health \& Care Excellence (NICE). Physical activity: exercise referral schemes. Public health guideline [PH54]. Published date: September 2014. www.nice.org.uk/ guidance/ph54 (accessed 7 June 2019).

\section{Noyes 2015}

Noyes J, Hendry M, Booth A, Lewin S, Glenton C, Garside R, et al. Guidance for review authors on choice and use of theory in complex intervention reviews. Version 1. London (UK): Cochrane, 2015.

\section{Noyes 2018}

Noyes J, Booth A, Lewin S, Carlsen B, Glenton C, Colvin CJ, et al. Applying GRADE-CERQual to qualitative evidence synthesis findings - paper 6: how to assess relevance of the data. Implementation Science 2018;13(Suppl 1):4. [DOI: 10.1186/ s13012-017-0693-6]

Factors that influence participation in physical activity for people with bipolar disorder: a synthesis of qualitative evidence (Protocol) 


\section{Orji 2012}

Orji R, Vassileva J, Mandryk R. Towards an effective health interventions design: an extension of the Health Belief Model. Online Journal of Public Health Informatics 2012;4(3):1-31. [DOI: 10.5210/ojphi.v4i3.4321; PMC3615835; PUBMED: 2356965]

\section{Osborn 2007}

Osborn D, Levy G, Nazareth I, Petersen I, Islam A, King MB. Relative risk of cardiovascular and cancer mortality in people with severe mental illness from the United Kingdom's General Practice Research Database. Archives of General Psychiatry 2007;64(2):242-9. [DOI: 10.1001/archpsyc.64.2.242]

\section{Patton 2002}

Patton MQ. Qualitative Research \& Evaluation Methods. 3rd Edition. Thousand Oaks, CA: Sage Publications, 2002.

\section{Peluso 2005}

Peluso MA, Andrade LH. Physical activity and mental health: the association between exercise and mood. Clinics 2005;60(1):61-70. [DOI: 10.1590/S1807-59322005000100012]

\section{Penedo 2005}

Penedo FJ, Dahn JR. Exercise and well-being: a review of mental and physical health benefits associated with physical activity. Current Opinion in Psychiatry 2005;18(2):189-93.

\section{Post 2013}

Post RM, Kalivas P. Bipolar disorder and substance misuse: pathological and therapeutic implications of their comorbidity and cross-sensitisation. British Journal of Psychiatry 2013;202(3):172-6. [DOI: 10.1192/bjp.bp.112.116855]

\section{Price 2018}

Price N, Williams N, Horton E, Liguori G, Mann S, Jimenez A. Effects of exercise referral schemes on physical activity levels. Medicine \& Science in Sports \& Exercise 2018;50(5S):48. [DOI: 10.1249/01.mss.0000535240.84785.24]

\section{Rehm 2019}

Rehm J, Shield KD. Global burden of disease and the impact of mental and addictive disorders. Current Psychiatry Reports 2019;21(2):10. [DOI: 10.1007/s11920-019-0997-0]

\section{Rosenbaum 2015}

Rosenbaum S, Sherrington C, Tiedemann A. Exercise augmentation compared with usual care for post-traumatic stress disorder: a randomized controlled trial. Acta Psychiatrica Scandinavica 2015;131(5):350-9. [DOI: 10.1111/acps.12371]

\section{Rosenstock 1960}

Rosenstock IM. What research in motivation suggests for public health. American Journal of Public Health and the Nation's Health 1960;50:295-302. [DOI: 10.2105/AJPH.50.3_Pt_1.295]

\section{Rosenstock 1974}

Rosenstock IM. Historical origins of the Health Belief Model. Health Education Monographs 1974;2(4):328-35. [DOI: 10.1177/109019817400200403]

\section{Roshanaei-Moghaddam 2009}

Roshanaei-Moghaddam B, Katon W. Premature mortality from general medical illnesses among persons with bipolar disorder: a review. Psychiatric Services 2009;60(2):147-56. [DOI: 10.1176/ ps.2009.60.2.147]

\section{Rowland 2018}

Rowland TA, Marwaha S. Epidemiology and risk factors for bipolar disorder. Therapeutic Advances in Psychopharmacology 2018;8(9):251-69. [DOI: 10.1177/2045125318769235]

\section{Schuch 2016}

Schuch FB, Dunn AL, Kanitz AC, Delevatti RS, Fleck MP. Moderators of response in exercise treatment for depression: a systematic review. Journal of Affective Disorders 2016;195:40-9. [DOI: 10.1016/j.jad.2016.01.014; PUBMED: 26854964]

\section{Schuch 2018}

Schuch FB, Vancampfort D, Firth J, Rosenbaum S, Ward PB, Silva ES, et al. Physical activity and incident depression: a meta-analysis of prospective cohort studies. American Journal of Psychiatry 2018;175(7):631-48. [DOI: 10.1176/ appi.ajp.2018.17111194; PUBMED: 29690792]

\section{Searle 2012}

Searle A, Calnan M, Turner KM, Lawlor DA, Campbell J, Chalder M, et al. General practitioners' beliefs about physical activity for managing depression in primary care. Mental Health and Physical Activity 2012;5(1):13-9. [EMBASE: 10.1016/ j.mhpa.2011.11.001]

\section{Searle 2014}

Searle A, Haase AM, Chalder M, Fox KR, Taylor AH, Lewis G, et al. Participants' experiences of facilitated physical activity for the management of depression in primary car. Journal of Health Psychology 2014;19(11):1430-42. [DOI: 10.1177/1359105313493648; PUBMED: 24021723]

\section{Shao 2018}

Shao C, Wang J, Liu J, Tian F, Li H. Effect of a Health Belief Model-based education program on patients' belief, physical activity, and serum uric acid: a randomized controlled trial. Patient Preference and Adherence 2018;12:1239-45. [DOI: https://doi.org/10.2147/PPA.S166523]

\section{Steinberg 1985}

Steinberg H, Sykes EA. Introduction to symposium on endorphins and behavioural processes; review of literature on endorphins and exercise. Pharmacology Biochemistry \& Behavior 1985;23(5):857-62. [PUBMED: 3909168]

\section{Strid 2016}

Strid C, Andersson C, Forsell Y, Öjehagen A, Lundh LG. Internetbased cognitive behaviour therapy and physical exercise effects - effects studied by automated telephone assessments in mental ill-health patients; a randomised controlled trial. British Journal of Clinical Psychology 2016;55(4):414-28. [DOI: 10.1111/ bjc.12111] 


\section{Ströhle 2009}

Ströhle A. Physical activity, exercise, depression and anxiety disorders. Journal of Neural Transmission 2009;116(6):777-84. [DOI: 10.1007/s00702-008-0092-x]

\section{Stubbs 2018}

Stubbs B, Vancampfort D, Hallgren M, Firth J, Veronese N, Solmi M, et al. EPA guidance on physical activity as a treatment for severe mental illness: a meta-review of the evidence and Position Statement from the European Psychiatric Association (EPA), supported by the International Organization of Physical Therapists in Mental Health (IOPTMH). European Psychiatry 2018;54:124-44. [DOI: 10.1016/j.eurpsy.2018.07.004]

\section{Sylvia 2011}

Sylvia LG, Nierenberg AA, Stange JP, Peckham AD, Deckersbach T. Development of an integrated psychosocial treatment to address the medical burden associated with bipolar disorder. Journal of Psychiatric Practice 2011;17(3):224-32. [DOI: 10.1097/01.pra.0000398419.82362.32; PMC3659403; PUBMED: 21587004]

\section{Sylvia 2013a}

Sylvia LG, Friedman ES, Kocsis JH, Berstein EE, Brody BD, Kinrys $\mathrm{G}$, et al. Association of exercise with quality of life and mood symptoms in a comparative effectiveness study of bipolar disorder. Journal of Affective Disorders 2013;151(2):722-7. [DOI: 10.1016/j.jad.2013.07.031]

\section{Sylvia 2013b}

Sylvia LG, Salcedo S, Bernstein EE, Baek JH, Nierenberg AA, Deckersbach T. Nutrition, exercise, and wellness treatment in bipolar disorder: proof of concept for a consolidated intervention. International Journal of Bipolar Disorders 2013;1(1):24. [DOI: 10.1186/2194-7511-1-24]

\section{Thomas 2008}

Thomas J, Harden A. Methods for the thematic synthesis of qualitative research in systematic reviews. BMC Medical Research Methodology 2008;8:45. [DOI: 10.1186/1471-2288-8-45]

\section{Thomson 2015}

Thomson D, Turner A, Lauder S, Gigler ME, Berk L, Singh AB, et al. A brief review of exercise, bipolar disorder and mechanistic pathways. Frontiers in Psychology 2015;6:147. [DOI: 10.3389/ fpsyg.2015.00147]

\section{Tidemalm 2008}

Tidemalm D, Waern M, Stefansson CG, Elofsson S, Runeson B. Excess mortality in persons with severe mental disorder in Sweden: a cohort study of 12,103 individuals with and without contact with psychiatric services. Clinical Practice and Epidemology in Mental Health 2008;4(23):1-9. [DOI: 10.1186/1745-0179-4-23; PMC2576252]

\section{Tsuchiya 2003}

Tsuchiya KJ, Byrne M, Mortensen PB. Risk factors in relation to an emergence of bipolar disorder: a systematic review. Bipolar Disorders 2003;5(4):231-42. [DOI: 10.1034/ j.1399-5618.2003.00038.x]

\section{US Dept of HHS 2008}

US Department of Health and Human Services. Physical Activity Guidelines Advisory Committee Report, 2008. health.gov/ paguidelines/2008/report/pdf/committeereport.pdf (accessed 24 January 2019).

\section{Vancampfort 2016}

Vancampfort D, Firth J, Schuch F, Rosenbaum S, De Hert M, Mugisha J, et al. Physical activity and sedentary behavior in people with bipolar disorder: a systematic review and metaanalysis. Journal of Affective Disorders 2016;201:145-52. [DOI: 10.1016/j.jad.2016.05.020]

\section{Vancampfort 2017}

Vancampfort D, Firth J, Schuch F, Rosenbaum S, Mugisha J, Hallgren $M$, et al. Sedentary behavior and physical activity levels in people with schizophrenia, bipolar disorder and major depressive disorder: a global systematic review and metaanalysis. World Psychiatry 2017;16(3):308-15. [DOI: 10.1002/ wps.20458]

\section{Wheeler 2018}

Wheeler AJ, Roennfeldt H, Slattery M, Krinks R, Stewart V. Codesigned recommendations for increasing engagement in structured physical activity for people with serious mental health problems in Australia. Health \& Social Care in the Community 2018;26(6):860-70. [DOI: 10.1111/hsc.12597; PUBMED: 30047608$]$

\section{WHO 1992}

World Health Organization. The ICD-10 classification of mental and behavioural disorders: clinical descriptions and diagnostic guidelines. Geneva: World Health Organization, 1992.

\section{WHO 2010}

World Health Organization. Global recommendations on physical activity for health. www.who.int/dietphysicalactivity/ publications/9789241599979/en/ (accessed 23 January 2019).

\section{WHO 2012}

World Health Organization. Recommended levels of physical activity for children aged 5-17 years. Global strategy on diet, physical activity and health. www.who.int/dietphysicalactivity/ factsheet_young_people/en/ (accessed 30 December 2019).

\section{WHO 2014}

World Health Organization. Social determinants of mental health. apps.who.int/iris/bitstream/ handle/10665/112828/9789?sequence=1 (accessed 23 January 2019).

\section{WHO 2018}

World Health Organization. WHO launches ACTIVE: a toolkit for countries to increase physical activity and reduce noncommunicable diseases. who.int/ncds/prevention/physicalactivity/active-toolkit/en/ (accessed 24 January 2019).

\section{Wilkinson 2018}

Wilkinson R, Pickett K. The Inner Level: How More Equal Societies Reduce Stress, Restore Sanity and Improve Everyone's Well-being. London: Penguin Books, 2018. 


\section{Wilmot 2012}

Wilmot EG, Edwardson CL, Achana FA, Davies MJ, Gorely LJ, Gray K, et al. Sedentary time in adults and the association with diabetes, cardiovascular disease and death: systematic review and meta-analysis. Diabetologia 2012;55(11):2895-905.

\section{Wright 2012}

Wright K, Armstrong T, Taylor A, Dean S. 'It's a double edged sword': a qualitative analysis of the experiences of exercise amongst people with Bipolar Disorder. Journal of Affective Disorders 2012;136(3):634-42. [DOI: 10.1016/j.jad.2011.10.017; PUBMED: 22100131]

\section{Wu 2016}

Wu MK, Wang HY, Chen YW, Lin PY, Wu CK, Tseng PT. Significantly higher prevalence rate of asthma and bipolar disorder co-morbidity: a meta-analysis and review under PRISMA
Guidelines. Medicine (Baltimore) 2016;95(13):e3217. [DOI: 10.1097/MD.0000000000003217; PMC4998549; PUBMED: 27043688]

\section{Wynanden 2012}

Wynaden D, Barr L, Omari O, Fulton A. Evaluation of service users' experiences of participating in an exercise programme at the Western Australian State Forensic Mental Health Services. International Journal of Mental Health Nursing 2012;21(3):229-35. [DOI: 10.1111/j.1447-0349.2011.00787.x; PUBMED: 22533330]

\section{Young 2017}

Young SJ, Praskova A, Hayward N, Patterson S. Attending to physical health in mental health services in Australia: a qualitative study of service users' experiences and expectations. Health \& Social Care in the Community 2017;25(2):602-11. [DOI: 10.1111/hsc.12349; PUBMED: 27093882]

\section{AP PEN DICES}

\section{Appendix 1. MEDLINE search strategy}

\section{Ovid MEDLINE ${ }^{\circledR}$ and Epub Ahead of Print, In-Process \& Other Non-Indexed Citations and Daily (1946 onwards)}

\section{[Population]}

1. "bipolar and related disorders"/ or bipolar disorder/

2. (bipolar adj3 (affective or depressi* or disorder? or mania or manic or psychos`)).ti,ab,kf.

3. ((affective or depressive) adj2 psychos $\left.{ }^{\star}\right)$.ti,ab,kf.

4. ((mania or manic) adj2 (depressi* or disorder? or episode? or state?)).ti,ab,kf.

5. (rapid cycling or schizoaffective).ti,ab,kf.

6. (bipolar or mania).ti,kf.

7. mentally ill persons/

8. or/1-7

[Intervention (broad, top level terms)]

9. ((access ${ }^{\star}$ or attitude? or barrier? or obstacle? or facilitat ${ }^{\star}$ or motivat ${ }^{\star}$ or preference? or predict*) adj3 (exercis* or (physical* adj (activit* or fit* or health)) or games or sport?)).ti,ab,kf.

10. *Life Style/ or Healthy Lifestyle/ or *Health Behavior/ or Sedentary Behavior/

11. (health promotion or lifestyle intervention? or (promot* adj2 healthy)).ti,ab,kf,sh.

12. ${ }^{\star}$ Motivation/px

13. or/9-12

[Outcome]

14. exp Exercise/

15. Exercise Therapy/

16. Physical Exertion/

17. Physical Fitness/

18. Leisure Activities/

19. exp Recreation/

20. exp Sports/

21. (exercis* or games or sport? or sporting or ((leisure or recreation*) adj activ*)).ti,ab,kf.

22. (running or jogging or hopping or skipping or sprinting or park run? or treadmill? or treadmill?).ti,ab,kf.

23. (hiking or tramping or mountaineer $\left.{ }^{\star}\right)$.ti,ab,kf,hw.

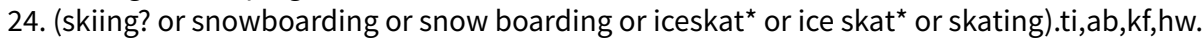

25. bicycling.sh. or (bike? or biking or bicycl* or ((recreational or distance) adj cycling)).ti,ab,kf.

26. (water sports or ((aqua* or water) adj (activit* or fit*)) or swimming or sailing or boating or yachting or canoeing or kayaking or surfing or sailboard* or sail board $\left.{ }^{\star}\right)$.ti,ab,kf,hw.

27. (team game? or football* or rugby or cricket or rounders or baseball or netball or volleyball or tennis or squash or badminton).ti,ab,kf,hw.

28. (physical ${ }^{*}$ adj (activit ${ }^{\star}$ or conditioning or training)).ti,ab,kf.

29. ((weight? adj1 (lift ${ }^{\star}$ or train $\left.{ }^{\star}\right)$ ) or ((strength or resistance) adj training)).ti,ab,kf.

30. exercise movement techniques/ or breathing exercises/ or qigong/ or dance therapy/ or tai ji/ or yoga/ or kinesiology/

31. (qigong or qi gong or ch'i kung or Tai Chi or Taiji or Tai Chi Chuan or Taichi Quan or Taijiquan or Shadowboxing or Shadow Boxing or Tai Chi Chih or T'ai Chi Chuan or yoga or yogic or pilates or kinesiology).ti,ab,kf,ot.

Factors that influence participation in physical activity for people with bipolar disorder: a synthesis of qualitative evidence (Protocol) 


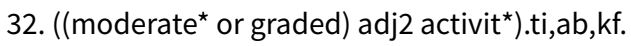

33. (aerobics or keep* fit or fitness training).ti,ab,kf.

34. (walking or walks or pedomet ${ }^{\star}$ or fitbit? or fit bit? or ((fitness or activit $\left.{ }^{\star}\right)$ adj track $\left.{ }^{\star}\right)$ or (steps adj2 (count ${ }^{\star}$ or track $^{\star}$ or monitor $\left.\left.^{\star}\right)\right)$ ).ti,ab,kf.

35. (ballet or dance or dancing or salsa or zumba).ti,ab,kf.

36. (gardening or horticultur* ${ }^{\star}$ allotment* or ((nature or animal) adj2 therap*)).ti,ab,kf,hw.

37. Fitness Trackers/

38. "Physical Education and Training"/

39. Fitness Centers/

40. (gymnasium? or ((fitness or leisure or wellness) adj (cent* or facility or facilities))).ti,ab,kf.

41. ((activity adj2 (amount ${ }^{\star}$ or increas ${ }^{\star}$ or level? or measur ${ }^{\star}$ or monitor $\left.{ }^{\star}\right)$ ) not (drug activity or active drug?)).ti.

42. (health promotion or lifestyle intervention?).ti,ab,kf,sh.

43. or/14-42

44. 8 and 13

45.8 and 43

46.44 or 45

47. exp animals/ not humans.sh.

48. (mice or mouse or murine or rat or rats or rodent ${ }^{\star}$ ).ti,kf.

49. 46 not ( 47 or 48$)$

Appendix 2. Data extraction template

\section{Background information}

\section{Author}

\section{Micro context: population}

(any specific characteristics, perspectives or subgroups?)

\section{Micro context: setting}

(setting e.g. hospital, private provider, timeframe of interest)

\section{Micro context: place}

(geographical location, political system e.g. state-funded healthcare)

\section{Meso-context: intervention}

(description of intervention, recruitment, dose, duration, delivery)

\section{Macro-context}

(policy, political issues, social climate or legislation)

\section{Data collection methods}

(e.g. focus group, face to face interviews, observation)

\section{Theory of change/logic model}

\section{Data analysis methods}

(e.g. thematic analysis, grounded theory)

\section{Relevant quantitative findings}


(Continued)

\section{Purposive sampling category}

$\begin{aligned} & \text { Popula- Diagnosis Methods } \\ & \text { tion }\end{aligned}$
tion

\section{Participants}

\section{Sample size}

\section{Perspective}

Service users, carers, service providers, health professionals

$\begin{array}{lll}\begin{array}{l}\text { Service } \\ \text { users }\end{array} & \text { Carers } & \begin{array}{l}\text { Service } \\ \text { providers }\end{array}\end{array}$

\section{Modifying factors}

(for each participant category viewpoint: service user, carers, service providers, health professionals)

Age

Socio-economic status (SES)

Ethnicity

Place of residence

Occupation

Education

Social capital

\section{Primary diagnosis}

(list specific diagnosis)

\section{Co-morbidity}

\section{Severity}

\section{Length of condition}

\section{Perceived seriousness and susceptibility}

Consideration of future

Self-identity

Perceived importance

Concern for appearance

Other perceptions of seriousness and susceptibility 
(Continued)

\section{Threats}

Perceived susceptibility: accepting mental health diagnosis and physical health needs

Perceived severity of mental health symptoms

Perceived physical health limits

\section{Other threats}

\section{Expectations}

Perceived benefits of physical activity

Perceived barriers to physical activity

Perceived self-efficacy: motivation, self-esteem

Other expectations

\section{Cues to action}

Public health messages

\section{Professionals}

Support networks

Telemonitoring

Other

\section{Outcomes}

Participation in physical activity

\section{Additional themes}

\section{CONTRIBUTIONSOF AUTHORS}

CMCC and JY conducted the initial literature review to prepare the protocol. JB, RC, GD and SG advised on the scope of the protocol. JB liaised with DRILL to request funding support. CMcC drafted the protocol. PB, GD, JF, BS, MT, PW and CW gave feedback on the protocol.

\section{DECLARATIONSOF INTEREST}

Claire J McCartan: no conflicts of interest

Jade Yap: no conflicts of interest

Joseph Firth: is supported by a National Institute of Complementary Medicine (NICM) and Blackmores Institute Fellowship. Brendon Stubbs: no conflicts of interest 
Mark A Tully: no conflicts of interest

Paul Best: no conflicts of interest

Paul Webb: no conflicts of interest

Chris White: no conflicts of interest

Simon Gilbody: no conflicts of interest

Rachel Churchill: leads and has responsibility for Cochrane Common Mental Disorders, which has supported parts of the review process and is largely funded by a grant from the National Institute for Health Research (NIHR) in the UK.

Josefien Breedvelt: no conflicts of interest

Gavin Davidson: is employed as the Praxis Chair of Social Care at Queen's University Belfast. This position is part funded by Praxis Care, a mental health service provider.

\section{SOURCES OF SUPPORT}

\section{Internal sources}

- Queen's University Belfast, UK.

- University of York, UK.

\section{External sources}

- Disability Research on Independent Living and Learning (DRILL), UK.

Funding was provided for CMcC's salary for 0.5 days per week over a three-month period to help complete this protocol.

- National Institute of Complementary Medicine (NICM) and Blackmores Institute, Australia.

JF is supported by a NICM-Blackmores Institute Fellowship.

- Praxis Care, UK.

GD's post at Queen's University Belfast is part funded by Praxis Care. 